\title{
Preoperative risk stratification using plasma fibrinogen levels can predict lymphovascular invasion and poor prognosis in patients with upper urinary tract urothelial carcinoma
}

\author{
KENJI KURODA, SHINSUKE TASAKI, JUNICHI ASAKUMA, AKIO HORIGUCHI and KEIICHI ITO \\ Department of Urology, National Defense Medical College, Tokorozawa, Saitama 359-8513, Japan
}

Received July 11, 2020; Accepted February 5, 2021

DOI: $10.3892 / \operatorname{mco} .2021 .2264$

\begin{abstract}
It has been previously indicated that preoperative plasma fibrinogen levels can correlate with cancer progression and be used as a useful predictor of lymph node metastasis or its premetastatic status such as lymphovascular invasion (LVI). In the present study, how preoperative plasma fibrinogen levels, considered in conjunction with other clinicopathological factors, can predict the presence of LVI and prognosisin patients with upper urinary tract urothelial carcinoma (UTUC) was examined. Medical records of 145 patients with UTUC who underwent radical nephroureterectomy (RNU) were retrospectively reviewed. The current study evaluated systemic inflammatory response markers including levels of plasma fibrinogen and other clinicopathological factors in orderto determine independent predictors of LVI and prognosis. The Cox proportional hazards model indicated that positive surgical margins and LVI were independent factors for poor cancer-specific survival (CSS) rates and extraurothelial recurrence-free survival (ERFS) rates. In addition, positive cytology, the presence of hydronephrosis and plasma fibrinogen levels were significant preoperative predictors of LVI. Furthermore, patients exhibiting two or more of higher fibrinogen levels ( $\geq 400 \mathrm{mg} / \mathrm{dl}$ ), positive urine cytology and the presence of hydronephrosis were indicated to exhibit worse CSS or ERFS rates compared with patients exhibiting only one of the aforementioned factors or those with none of the three aforementioned factors in the multivariate analysis of the Cox proportional hazards model. In conclusion, hyperfibrinogenemia can be an independent predictor of the presence of LVI, and stratifying preoperative risk using fibrinogen levels, urine cytology and hydronephrosis can serve as the basis for selecting candidates for additional therapy before and/or after RNU in patients with UTUC.
\end{abstract}

Correspondence to: Dr Kenji Kuroda, Department of Urology, National Defense Medical College, 3-2 Namiki, Tokorozawa, Saitama 359-8513, Japan

E-mail:kksmy@sa2.so-net.ne.jp

Key words: upper urinary tract urothelial carcinoma, fibrinogen, urine cytology, hydronephrosis, lymphovascular invasion

\section{Introduction}

Upper urinary tract urothelial carcinoma (UTUC) is a relatively rare malignant tumor, estimated to be approximately $10 \%$ of all renal tumors and only $5 \%$ of all urothelial carcinomas $(1,2)$. This corresponds to an estimated annual incidence of almost two cases per 100,000 inhabitants in Western countries.

Radical nephroureterectomy (RNU) with a bladder cuff excision is the standard treatment for patients with NOM0 UTUC; however, lymphovascular invasion (LVI) as well as tumor grade and pathological stage were significantly related to cancer-specific survival (CSS) and overall survival rates in patients with UTUC who underwent open or laparoscopic RNU (3). Based on these findings, appropriate markers to monitor or predict oncologic outcomes for patients with localized UTUC are necessary.

The prognostic value of many systemic inflammatory response (SIR) markers as predictors of poor CSS or recurrence-free survival in several types of carcinoma has been reported (4-6). SIR was found to be related to shorter time to cancer progression and cancer-specific death (4-6). SIR markers include preoperative $\mathrm{C}$-reactive protein (CRP), the Glasgow Prognostic Score (GPS), neutrophil to lymphocyte ratio (NLR), and platelet to lymphocyte ratio (PLR) (7-10).

Taken together, several studies have focused on the relationship between various cancers and hemostatic factors. Among such hemostatic factors, fibrinogen, an essential hemostatic factor, is converted to fibrin (a final product of the hemostatic pathway) by activated thrombin. In addition, elevated plasma fibrinogen has been reported to correlate with tumor progression in some types of cancer (11-13). Moreover, preoperative plasma fibrinogen levels were found to be a useful predictor of lymph node metastasis in earlier studies $(11,12)$. As a result, preoperative plasma fibrinogen levels were also relevant in terms of predicting a poor prognosis in patients with several types of cancer (14-16). Based on these studies, we hypothesize that fibrinogen could be one cause of distant metastasis in patients with UTUC. Furthermore, a higher plasma fibrinogen level may also be a feasible marker of premetastatic status such as LVI; therefore, this marker mayenable us to monitor or predict oncologic outcomes of patients with localized UTUC, considered in conjunction with other clinicopathological factors. In the present study, we retrospectively examined the preoperative plasma 
fibrinogen levels of patients with UTUC who underwent RNU and evaluated the association of clinicopathological risk factors including preoperative plasma fibrinogen levelswith the presence of LVI and CSS and ERFS rates to establish a preoperative risk stratification model.

\section{Patients and methods}

Patients. We retrospectively reviewed and analyzed clinicopathological data from the medical records of 145 patients who underwent RNU at our institution and who were histologically diagnosed with UTUC. The study protocol (ID 2734) was approvedon June 14, 2017, by our institutional ethics committee, and we used an opt-out approach on the Web page of the National Defense Medical College instead of collecting written informed consent from all participants. The patients included 109 men and 36 women. The median follow-up period after nephroureterectomy was 54.2 months (range: 3.4 to 209.2 months). We present additional clinicopathological data in Table I.

Extraurothelial recurrence after nephroureterectomy indicates tumor recurrence outside the bladder or distant metastasis. In this study, we defined recurrence-free survival as extraurothelial recurrence-free survival (ERFS). No patients had distant metastasis at diagnosis. Extraurothelialor intravesical recurrence was monitored for each patient every 3-6 months for the first 5 years after nephroureterectomy and 6-12 months thereafter. All surgical specimens were processed according to standard pathological procedures and were histologically confirmed to be urothelial carcinoma with or without other tumor cell types. The pathological staging of the primary tumor was determined according to the American Joint Committee on Cancer TNM Classification (17), whereas tumor grading was determined according to the 2004 World Health Organization (WHO) classification of urothelial tumors (18). Tumor specimens were evaluated by two pathologists, and the patients were categorized into two groups on the basis of the 2004 WHO classification system for tumor grading.

Inflammatory indices. Inflammatory indices were evaluated by laboratory tests. The neutrophil to lymphocyte ratio (NLR) was calculated by dividing the absolute neutrophil count by the absolute lymphocyte count (19). The platelet to lymphocyte ratio (PLR) was calculated by dividing the absolute platelet count by the absolute lymphocyte count (19). The Glasgow prognostic score (GPS) was evaluated by using the CRP and Alb values. Patients with elevated CRP levels $(>1 \mathrm{mg} / \mathrm{dl})$ in combination with hypoalbuminemia $(<3.5 \mathrm{~g} / \mathrm{dl})$ were allocated 2 points. Patients with elevated CRP alone or hypoalbuminemia alone were allocated 1 point, whereas patients exhibiting these parameters within normal limits were allocated 0 points (10). In the presents study, normal limits of serum albumin, CRP levels and plasma fibrinogen levels were designated according to analyzing kits used at our institution based on the methods shown in earlier reports $(11-13,20)$.

Statistical analysis. Cox proportional hazards model was used in the examination of independent factors for worse CSS and ERFS rates in multivariate analysis. A receiver operator characteristic (ROC) analysis was performed to determine the cut-off values of NLR and PLR according to the method shown in previous reports $(21,22)$. Fisher's exact probability test was performed to examine the relationship between clinicopathological factors and the presence of LVI. Multiple logistic regression analysis was performed to detect predictors of LVI. The effect of independent markers for worse CSS and ERFS rates was evaluated with Kaplan-Meier plots and the log-rank test. The statistical analysis was undertaken using JMP version 14 (SAS Institute, Inc.). $\mathrm{P}<0.05$ was considered to indicate a statistically significant difference.

\section{Results}

Independent factors for poor CSS rate and ERFS rate. The Cox proportional hazards model indicated that tumor histology, pathological $\mathrm{T}$ stage, tumor grade, lymph node metastasis, positive surgical margins, LVI, and the presence of hydronephrosis were independent factors for poor CSS and ERFS ratesin univariate analysis, and positive surgical margins and LVI were independent predictors of worse CSS $(\mathrm{P}=0.035, \mathrm{P}=0.001$, respectively) and ERFS $(\mathrm{P}<0.001$, $\mathrm{P}=0.003$, respectively) rates in multivariate analysis among pathological factors (Tables II and III). Finally, cancer-specific mortality and extraurothelial recurrence were found in 37 and 41 patients, respectively.

Association between clinicopathological factors and the presence of LVI. Out of these two pathological factors, LVI was expected to be more frequently seen in surgical specimens unless pathological $\mathrm{T}$ stage was equal to or higher than 3 . Therefore, we investigated the association between preoperative factors and the presence of LVI. Positive cytology, the presence of hydronephrosis, C-reactive protein levels, NLR, PLR, and plasma fibrinogen levels were significantly associated with the presence of LVI among preoperative factors in Fisher's exact probability test (Table IV). Among these related factors, positive cytology, the presence of hydronephrosis, and plasma fibrinogen levels were significant preoperative predictors of the presence of LVI $(\mathrm{P}=0.008, \mathrm{P}=0.010, \mathrm{P}<0.001$, respectively) (Table $\mathrm{V}$ ). In fact, patients with higher fibrinogen levels ( $\geq 400 \mathrm{mg} / \mathrm{dl}$ ) had worse CSS and ERFS rates (both $\mathrm{P}<0.001$ ) (Fig. 1). Patients with positive urine cytology did not have poor CSS or ERFS rates when compared with those with negative cytology ( $\mathrm{P}=0.319, \mathrm{P}=0.666$, respectively) (Fig. 2). Patients with hydronephrosis had worse CSS and ERFS rates than those without hydronephrosis $(\mathrm{P}=0.042, \mathrm{P}=0.019$, respectively) (Fig. 3).

\section{Preoperative risk stratification using plasma fibrinogen} levels and other risk factors to predict worse CSS and ERFS rates. Based on these findings, we stratified patients into three groups. Group A was comprised by patients exhibiting two or more of higher fibrinogen levels $(\geq 400 \mathrm{mg} / \mathrm{dl})$, positive urine cytology, and the presence of hydronephrosis. Group B consisted of patients with only one of those factors, and Group C consisted of those with none of the three factors. Group A patients showed worse CSS and ERFS rates than those in Groups B and C (both $\mathrm{P}<0.001$, Fig. 4). Moreover, we further divided patients into those with renal pelvic cancer 
Table I. Clinicopathological features.

\begin{tabular}{lr}
\hline Parameters & Patien \\
\hline Age (median 70) & 7 \\
$\geq 71$ & 74 \\
$\leq 70$ & \\
Sex & 109 \\
Male & 36 \\
Female & \\
Urine cytology & 88 \\
Positive & 57 \\
Negative & \\
Histology & \\
UC with other components & \\
UC alone & 11
\end{tabular}

Pathological T stage

$\geq \mathrm{T} 3$

$\leq \mathrm{T} 2$

Tumor grade

High

PUNLMP/Low

Lymph node metastasis

Positive

Negative

Ureteral involvement

Positive

Negative

Surgical margins

Positive

Negative

Lymphovascular invasion

Positive

Negative

Carcinoma in situ

Present

Absent

Hydronephrosis

Positive

Negative

CRP $(\geq 0.4$ or $\leq 0.3)$

$\geq 0.4$

$\leq 0.3$

Albumin ( $\leq 3.7$ or $\geq 3.8)$

$\leq 3.7$

$\geq 3.8$

NLR $(\geq 1.652$ or $<1.652)$

$\geq 1.652$

$<1.652$

PLR $(\geq 154.122$ or $<154.122)$

$\geq 154.122$

$<154.122$

GPS

$\geq 1$

0
Table I. Continued.

Parameters
Fibrinogen $(\geq 400$ or $<400)$
$\geq 400$
$<400$

Patients (n)

UC, urothelial carcinoma; PUNLMP, papillary urothelial neoplasm of low malignant potential; CRP, C-reactive protein; NLR, neutrophil to lymphocyte ratio; PLR, platelet to lymphocyte ratio; GPS, glasgow prognostic score.

alone and those with renal pelvic and/or ureteral cancer. Kaplan-Meier curves revealed that in both patients with renal pelvic cancer alone and those with renal pelvic and/or ureteral cancer, patients of Group A had significantly worse CSS $(\mathrm{P}=0.025, \mathrm{P}=0.018$, respectively) and ERFS $(\mathrm{P}=0.046$, $\mathrm{P}=0.039$, respectively) rates than those in Groups $\mathrm{B}$ and $\mathrm{C}$ (Figs. 5 and 6).

\section{Discussion}

In this study, a Cox proportional hazards model demonstrated that positive surgical margins and LVI were independent predictors of shorter CSS and ERFS time (Tables II and III). In addition, we found that fibrinogen levels, positive urine cytology, and the presence of hydronephrosis were significant factors associated with the presence of LVI (Tables IV and $\mathrm{V}$ ). In fact, patients exhibiting two or more of these factors showed worse CSS or ERFS rates than those exhibiting only one factor or those exhibiting none of the three factors (Fig. 4). These results were also consistent in patients with renal pelvic cancer alone and those with renal pelvic and/or ureteral canceras seen in all patients (Figs. 5 and 6). In addition, plasma fibrinogen levels are associated with other diseases (e.g., atherosclerosis, hypertension, and stroke). However, histories of such severe diseases were not found in patients enrolled in the present study. It is speculated that plasma fibrinogen levels were only predictors of worse CSS and ERFS in this study.

Several studies have indicated the association between elevated preoperative plasma fibrinogen levels and poor prognosis in a number of cancers by using meta-analyses (23-25). Earlier studies have also revealed that the metastatic status can potentiallyoccur in the environment of circulating tumor cells which may be caused by high plasma fibrinogen levels (26). Furthermore, preoperative plasma fibrinogen levels may berelated to tumor volume and may provide favorable conditions for circulating tumor cells to metastasize through the lymphatic drainage orvascular flow $(12,23)$. A preliminary study showed that mice lacking fibrinogen had a remarkably decreased level of lymphatic and hematogenous metastases compared to that observed in wild-type mice. They suggested that an increase in plasma fibrinogen levels can play an important role in cancer metastasis (26). Tanaka et al especially showed that patients with malignant tumors and preoperative high plasma fibrinogen levels have a higher risk for a worse prognosis than those with low fibrinogen levels in patients with 
Table II. Univariate and multivariate analyses of independent factors for cancer-specific survival.

\begin{tabular}{|c|c|c|c|c|c|c|c|}
\hline \multirow[b]{2}{*}{ Pathological parameters } & \multicolumn{2}{|c|}{ Univariate } & \multicolumn{5}{|c|}{ Multivariate } \\
\hline & HR & P-value & HR & & $95 \%$ & & P-value \\
\hline $\operatorname{Age}(\geq 71$ or $\leq 70)$ & 1.436 & 0.272 & & & & & \\
\hline Sex (male or female) & 0.836 & 0.623 & & & & & \\
\hline Urine cytology (positive or negative) & 1.416 & 0.313 & & & & & \\
\hline Tumor histology (UC with other components or UC alone) & 3.099 & 0.003 & 1.762 & 0.738 & - & 3.969 & 0.195 \\
\hline Pathological $\mathrm{T}$ stage $(\geq \mathrm{T} 3$ or $\leq \mathrm{T} 2$ ) & 4.275 & $<0.001$ & 1.624 & 0.710 & - & 4.017 & 0.258 \\
\hline Tumor grade (high or PUNLMP/low) & 4.824 & $<0.001$ & 2.549 & 0.926 & - & 9.012 & 0.072 \\
\hline Lymph node metastasis (positive or negative) & 4.320 & 0.006 & 0.720 & 0.230 & - & 2.029 & 0.544 \\
\hline Ureter involvement (positive or negative) & 1.652 & 0.143 & & & & & \\
\hline Surgical margins (positive or negative) & 3.281 & 0.003 & 2.388 & 1.064 & - & 5.083 & 0.035 \\
\hline Lymphovascular invasion (positive or negative) & 7.570 & $<0.001$ & 3.965 & 1.688 & - & 9.903 & 0.001 \\
\hline Carcinoma in situ (positive or negative) & 0.582 & 0.333 & & & & & \\
\hline Hydronephrosis (positive or negative) & 2.144 & 0.035 & 1.227 & 0.562 & - & 2.903 & 0.618 \\
\hline
\end{tabular}

HR, hazard ratio; CI, confidence interval; UC, urothelial carcinoma; PUNLMP, papillary urothelial neoplasm of low malignant potential.

Table III. Univariate and multivariate analyses of independent factors for recurrence-free survival.

\begin{tabular}{|c|c|c|c|c|c|c|c|}
\hline \multirow[b]{2}{*}{ Pathological parameters } & \multicolumn{2}{|c|}{ Univariate } & \multicolumn{5}{|c|}{ Multivariate } \\
\hline & HR & P-value & HR & & $95 \%$ & & P-value \\
\hline Age $(\geq 71$ or $\leq 70)$ & 1.499 & 0.197 & & & & & \\
\hline Sex (men or women) & 0.698 & 0.295 & & & & & \\
\hline Urine cytology (positive or negative) & 1.150 & 0.664 & & & & & \\
\hline Tumor histology (UC with other components or UC alone) & 3.625 & $<0.001$ & 2.178 & 0.993 & - & 4.643 & 0.052 \\
\hline Pathological $\mathrm{T}$ stage $(\geq \mathrm{T} 3$ or $\leq \mathrm{T} 2)$ & 4.461 & $<0.001$ & 2.093 & 0.965 & - & 4.859 & 0.062 \\
\hline Tumor grade (high or PUNLMP/low) & 5.426 & $<0.001$ & 3.182 & 1.162 & - & 11.223 & 0.023 \\
\hline Lymph node metastasis (positive or negative) & 6.713 & $<0.001$ & 1.338 & 0.495 & - & 3.283 & 0.547 \\
\hline Ureter involvement (positive or negative) & 1.751 & 0.086 & & & & & \\
\hline Surgical margins (positive or negative) & 3776 & $<0.001$ & 5.102 & 2.318 & - & 10.833 & $<0.001$ \\
\hline Lymphovascular invasion (positive or negative) & 6.822 & $<0.001$ & 3.147 & 1.480 & - & 7.000 & 0.003 \\
\hline Carcinoma in situ (positive or negative) & 0.541 & 0.263 & & & & & \\
\hline Hydronephrosis (positive or negative) & 2.288 & 0.016 & 1.438 & 0.685 & - & 3.240 & 0.345 \\
\hline
\end{tabular}

HR, hazard ratio; CI, confidence interval; UC, urothelial carcinoma; PUNLMP, papillary urothelial neoplasm of low malignant potential.

UTUC (20). Hence, the results of the present study concur with the results reported by Tanaka et al and others.

The prognostic value of chronic kidney disease and positive surgical margins in UTUC patients undergoing RNU was previously reported (27). However, preoperative plasma fibrinogen levels were not independent predictors of worse CSS or ERFS because the patient group of the previous study was different from that of the present study. In addition, the cut-off value of plasma fibrinogen levels also differed from that used in the present study (27).

Questions remain on how fibrinogen can influence the progression and metastatic possibility ofmalignant tumors. Several tentative theories have been reported on this. First, fibrinogen may aggregate around cancer cells to work as a structure for cancer cell proliferation. Fibrinogen can also serve as a basement to sustain some growth factors, such as vascular endothelial growth factor and fibroblast growth factor, to promote tumor angiogenesis (25). Second, tumor cells retain fibrinogen receptors, which play a role in bridging fibrinogen molecules to tumor cells and thus enhance the endothelial adhesion of tumor cell emboli in a target organ's vasculature. This mechanism appears to enable metastasis and successively increase the survival rates of metastatic cells (24). Third, fibrinogen can enhance the adhesion of platelets to tumor cells, mediated by $\beta 3$-integrin which was expressed by tumor cells. The aggregation of fibrinogen can be proceeded by platelet migration to the tumor cell environment; a thick layer of fibrin may then be formed to 
Table IV. Association between preoperative parameters and LVI.

A, Clinicopathological parameters

\begin{tabular}{|c|c|c|c|c|c|c|c|}
\hline \multirow[b]{2}{*}{ Parameter } & \multirow[b]{2}{*}{ Total } & \multirow[b]{2}{*}{$(\%)$} & \multicolumn{4}{|c|}{ LVI } & \multirow[b]{2}{*}{ P-value } \\
\hline & & & Positive & $(\%)$ & Negative & $(\%)$ & \\
\hline Age (median 70) & & & & & & & 0.492 \\
\hline$\geq 71$ & 71 & (49.0) & 23 & (32.4) & 48 & $(67.6)$ & \\
\hline$\leq 70$ & 74 & $(51.0)$ & 28 & $(37.8)$ & 46 & $(62.2)$ & \\
\hline Sex & & & & & & & 0.085 \\
\hline Male & 109 & $(75.2)$ & 34 & $(31.2)$ & 75 & $(68.8)$ & \\
\hline Female & 36 & $(24.8)$ & 17 & $(47.2)$ & 19 & $(52.8)$ & \\
\hline Urine cytology & & & & & & & 0.029 \\
\hline Positive & 88 & $(60.7)$ & 37 & $(42.1)$ & 51 & $(57.9)$ & \\
\hline Negative & 57 & (39.3) & 14 & (24.6) & 43 & $(75.4)$ & \\
\hline Hydronephrosis & & & & & & & 0.005 \\
\hline Positive & 86 & $(59.3)$ & 38 & $(44.2)$ & 48 & $(55.8)$ & \\
\hline Negative & 59 & (40.7) & 13 & $(22.0)$ & 46 & $(78.0)$ & \\
\hline
\end{tabular}

B, Laboratory parameters

LVI

\begin{tabular}{|c|c|c|c|c|c|c|c|}
\hline \multirow{2}{*}{ Parameter } & \multirow[b]{2}{*}{ Total } & \multirow[b]{2}{*}{ (\%) } & & & & & \multirow[b]{2}{*}{ P-value } \\
\hline & & & Positive & $(\%)$ & Negative & $(\%)$ & \\
\hline \multicolumn{8}{|l|}{ Laboratory parameters } \\
\hline \multicolumn{7}{|l|}{$\operatorname{CRP}(\geq 0.4$ or $\leq 0.3)$} & 0.015 \\
\hline$\geq 0.4$ & 34 & (23.5) & 18 & (52.9) & 16 & $(47.1)$ & \\
\hline$\leq 0.3$ & 111 & $(76.5)$ & 33 & (29.7) & 78 & $(70.3)$ & \\
\hline \multicolumn{7}{|l|}{ Albumin $(\leq 3.7$ or $\geq 3.8$ ) } & 0.110 \\
\hline$\leq 3.7$ & 17 & $(11.7)$ & 9 & (52.9) & 8 & $(47.1)$ & \\
\hline$\geq 3.8$ & 128 & $(88.3)$ & 42 & $(32.8)$ & 86 & $(67.2)$ & \\
\hline \multicolumn{7}{|l|}{ NLR $(\geq 1.652$ or $<1.652)$} & $<0.001$ \\
\hline$\geq 1.652$ & 114 & $(78.6)$ & 48 & $(42.1)$ & 66 & $(57.9)$ & \\
\hline$<1.652$ & 31 & $(21.4)$ & 3 & $(9.7)$ & 28 & $(90.3)$ & \\
\hline \multicolumn{7}{|l|}{$\operatorname{PLR}(\geq 154.122$ or $<154.122)$} & 0.005 \\
\hline$\geq 154.122$ & 60 & $(41.4)$ & 29 & $(48.3)$ & 31 & (51.7) & \\
\hline$<154.122$ & 85 & $(58.6)$ & 22 & $(25.9)$ & 63 & $(74.1)$ & \\
\hline \multicolumn{7}{|l|}{ GPS ( $\geq 1$ or 0$)$} & 0.128 \\
\hline$\geq 1$ & 15 & $(10.3)$ & 8 & (53.3) & 7 & $(46.7)$ & \\
\hline 0 & 130 & $(89.7)$ & 43 & $(33.1)$ & 87 & $(66.9)$ & \\
\hline \multicolumn{7}{|l|}{ Fibrinogen ( $\geq 400$ or $<400$ ) } & $<0.001$ \\
\hline$\geq 400$ & 23 & $(15.9)$ & 19 & (82.6) & 4 & (17.4) & \\
\hline$<400$ & 122 & $(84.1)$ & 32 & $(26.2)$ & 90 & $(73.8)$ & \\
\hline
\end{tabular}

LVI, lymphovascular invasion; CRP, C-reactive protein; NLR, neutrophil to lymphocyte ratio; PLR, platelet to lymphocyte ratio; GPS, glasgow prognostic score.

provide protection of the tumor cells against natural killer cell cytotoxicity, resulting in a higher possibility of distant metastasis (28). Moreover, fibrinogen can be integrated by cancer cells endogenously. This event can lead to the proliferation of cancer cells and angiogenesis, caused by fibroblast growth factor-2, which induces cancer progression and metastasis (29).

Positive urine cytology and the presence of hydronephrosis have been associated with the presence of LVI. Sakano et al showed that positive urine cytology and hydronephrosis 
Table V. Univariate and multivariate analyses of preoperative factors for the presence of LVI.

\begin{tabular}{|c|c|c|c|c|c|c|c|}
\hline \multirow{3}{*}{$\begin{array}{l}\text { Preoperative parameters } \\
\text { Urine cytology (positive or negative) }\end{array}$} & \multicolumn{2}{|c|}{ Univariate } & \multicolumn{5}{|c|}{ Multivariate } \\
\hline & \multirow{2}{*}{$\frac{\mathrm{OR}}{2.228}$} & \multirow{2}{*}{$\frac{\text { P-value }}{0.033}$} & \multirow{2}{*}{$\begin{array}{c}\text { OR } \\
3.439\end{array}$} & \multicolumn{3}{|c|}{$95 \% \mathrm{CI}$} & \multirow{2}{*}{$\frac{\text { P-value }}{0.008}$} \\
\hline & & & & 1.373 & - & 8.613 & \\
\hline Hydronephrosis (present or absent) & 2.801 & 0.007 & 3.398 & 1.334 & - & 8.654 & 0.010 \\
\hline $\mathrm{CRP}(\geq 0.4$ or $\leq 0.3)$ & 2.659 & 0.015 & 0.842 & 0.286 & - & 2.479 & 0.756 \\
\hline Albumin $(\leq 3.7$ or $\geq 3.8)$ & 2.304 & 0.110 & & & & & \\
\hline NLR $(\geq 1.652$ or $<1.652)$ & 6.788 & 0.003 & 4.158 & 0.971 & - & 17.794 & 0.055 \\
\hline $\operatorname{PLR}(\geq 154.122$ or $<154.122)$ & 2.679 & 0.006 & 1.131 & 0.479 & - & 2.673 & 0.778 \\
\hline GPS $(\geq 1$ or 0$)$ & 2.312 & 0.128 & & & & & \\
\hline Fibrinogen $(\geq 400$ or $<400)$ & 13.359 & $<0.001$ & 14.702 & 3.799 & - & 56.900 & $<0.001$ \\
\hline
\end{tabular}

LVI, lymphovascular invasion; OR, odds ratio; CI, confidence interval; CRP, C-reactive protein; NLR, neutrophil to lymphocyte ratio; PLR, platelet to lymphocyte ratio; GPS, glasgow prognostic score.
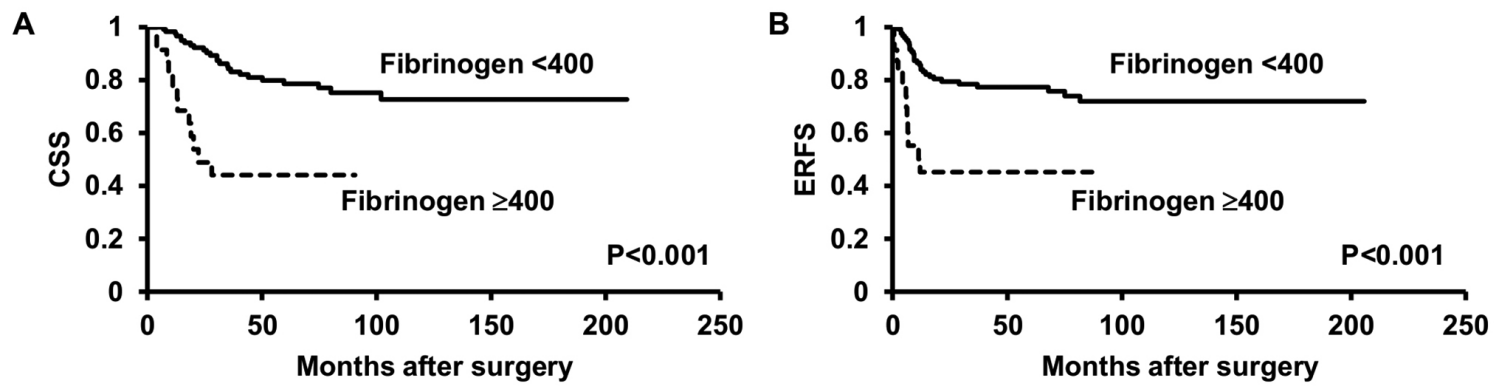

Figure 1. Survival analysis based on plasma fibrinogen levels in all patients. (A) CSS time in all patients with UTUC with or without plasma fibrinogen levels $\geq 400$ ( $\mathrm{P}<0.001)$. (B) ERFS time in all patients with UTUC with or without plasma fibrinogen levels $\geq 400(\mathrm{P}<0.001)$. CSS, cancer-specific survival; UTUC, urinary tract urothelial carcinoma; ERFS, extraurothelial recurrence-free survival.
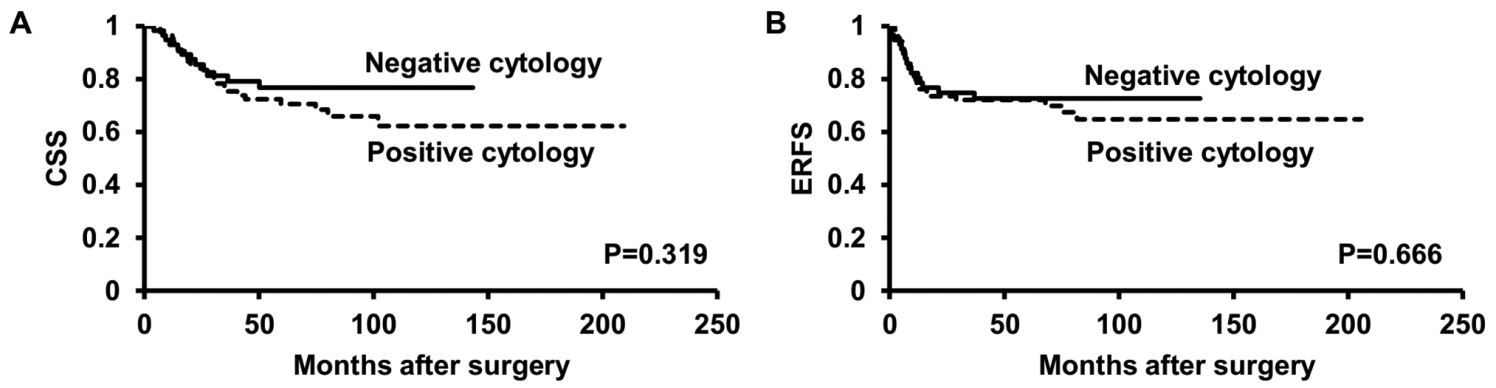

Figure 2. Survival analysis based on urine cytology in all patients. (A) CSS time in all patients with UTUC with or without positive urine cytology (P=0.319) (B) ERFS time in all patients with UTUC with or without positive urine cytology $(\mathrm{P}=0.666)$. CSS, cancer-specific survival; UTUC, urinary tract urothelial carcinoma; ERFS, extraurothelial recurrence-free survival.

were significantly associated with the presence of LVI (30). Ito et al also indicated that a higher hydronephrosis grade had a significant correlation with the presence of ureteral tumors, a higher pT stage, and the presence of LVI (31). Another study also showed that patients with positive voided urine cytology had higher incidences of high-grade tumors and positive LVI in UTUC patients treated with RNU (32). These findings are consistent with those of the present study, although neither positive urine cytology nor hydronephrosis was found as an independent factor for worse CSS or ERFS rates. However, using plasma fibrinogen levels in conjunction with these clinicopathological factors couldmore accurately predict LVI and a poorer prognosis in UTUC, as described in an earlier study evaluating the effectiveness of a two-factor risk stratification model (33).

This study had some limitations. First, it was retrospective in nature, and our sample size was relatively small; however, the median follow-up period after RNU was more than four years, and so our observation time to cancer-specific death and extraurothelial recurrence was long enough to support our findings. Second, the underlying mechanism explaining the association of positive urine cytology or hydronephrosis 
A

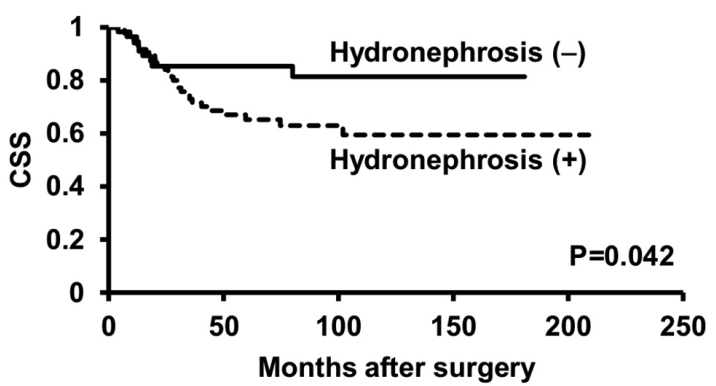

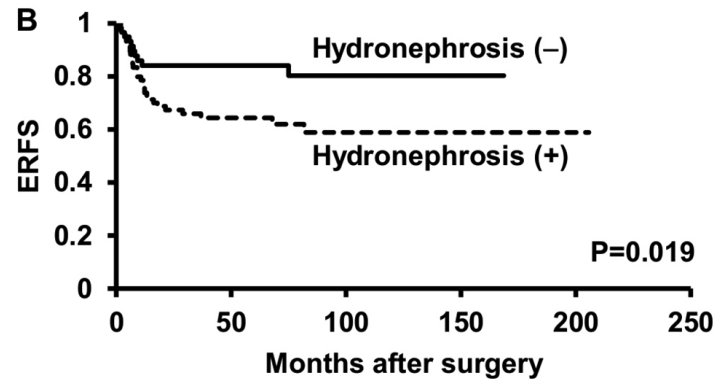

Figure 3. Survival analysis based on the presence of hydronephrosis in all patients. (A) CSS time in all patients with UTUC with or without hydronephrosis $(\mathrm{P}=0.042)(\mathrm{B})$ ERFS time in all patients with UTUC with or without hydronephrosis $(\mathrm{P}=0.019)$. CSS, cancer-specific survival; UTUC, urinary tract urothelial carcinoma; ERFS, extraurothelial recurrence-free survival.
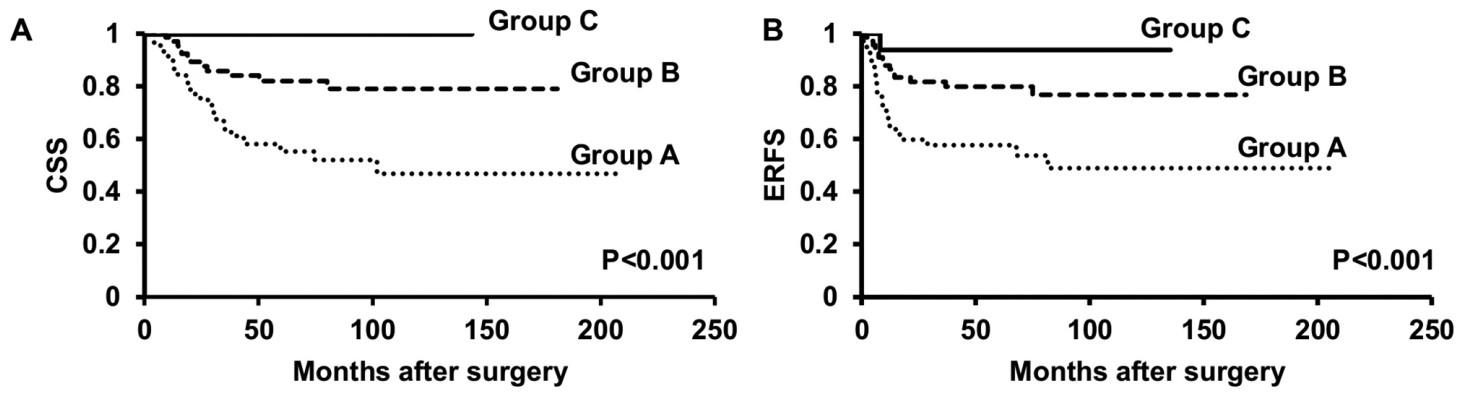

Figure 4. Survival analysis based on risk factors in all patients. (A) CSS time in all patients with UTUC among Groups A-C (P<0.001) (B) ERFS time in all patients with UTUC among Groups A-C ( $<<0.001)$. Group A: Patients with two or more of higher fibrinogen levels $(\geq 400 \mathrm{mg} / \mathrm{dl})$, positive urine cytology, and hydronephrosis; Group B: Patients with only one of these factors; and Group C: Patients with none of the abovementioned factors. CSS, cancer-specific survival; UTUC, urinary tract urothelial carcinoma; ERFS, extraurothelial recurrence-free survival.
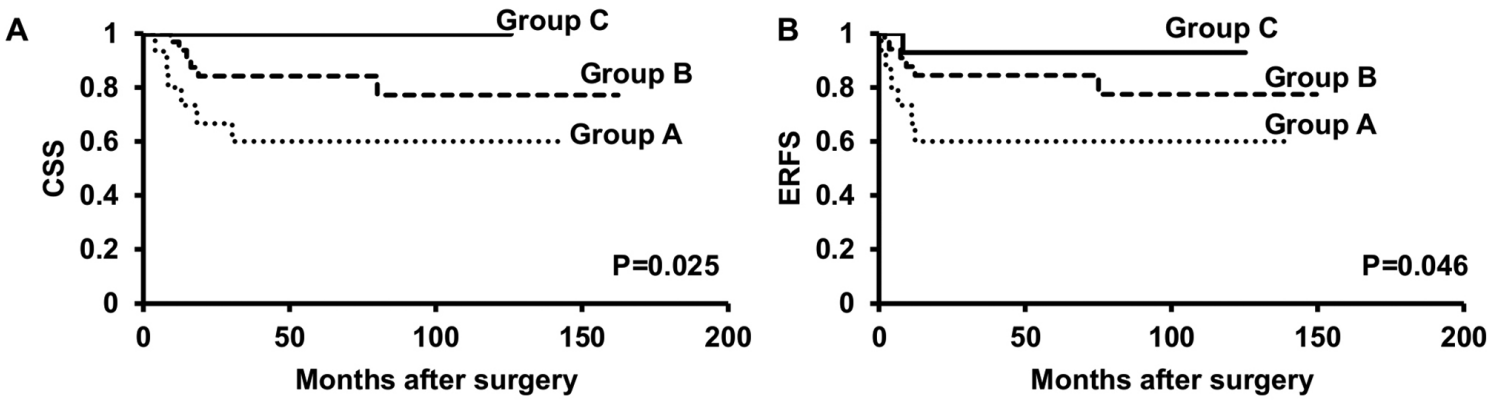

Figure 5. Survival analysis based on risk factors in patients with renal pelvic cancer alone. (A) CSS time in patients with renal pelvic cancer alone among Groups A-C $(\mathrm{P}=0.025)$ (B) ERFS time in patients with renal pelvic cancer alone among Groups A-C ( $\mathrm{P}=0.046)$. Group A: Patients with two or more of higher fibrinogen levels ( $\geq 400 \mathrm{mg} / \mathrm{dl}$ ), positive urine cytology, and hydronephrosis; Group B: Patients with only one of these factors; and Group C: Patients with none of the abovementioned factors. CSS, cancer-specific survival; ERFS, extraurothelial recurrence-free survival.
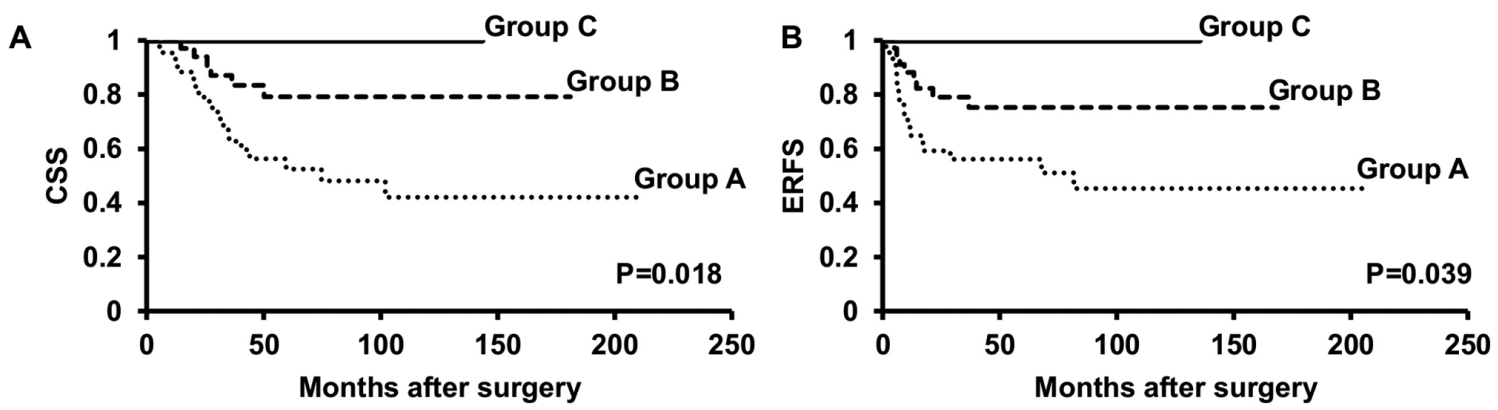

Figure 6. Survival analysis based on risk factors in patients with renal pelvic and/or ureteral cancer. (A) CSS time in patients with renal pelvic and/or ureteral cancer among Groups A-C ( $\mathrm{P}=0.018)$ (B) ERFS time in patients with renal pelvic and/or ureteral cancer among Groups A-C ( $\mathrm{P}=0.039)$. Group A: Patients with two or more of higher fibrinogen levels ( $\geq 400 \mathrm{mg} / \mathrm{dl}$ ), positive urine cytology, and hydronephrosis; Group B: Patients with only one of these factors; and Group C: Patients with none of the abovementioned factors. CSS, cancer-specific survival; ERFS, extraurothelial recurrence-free survival. 
with the presence of LVI remains unclear. As to hydronephrosis, its presence may imply tumoral extension to the outer urothelial layers, which may be an indicator of cancer cells spreading to the reginal nodes or distant organs. Considering that the urothelial lumen under hydronephrosis is thinner than normal lumen, the spreading of urothelial carcinoma cells beyond the urothelial layers is possible. The increased pressure in the urothelial lumen may also lead to counterflow in lymphatics and blood vessels, increasing the possibility of cancer cell migration (34). Furthermore, we could not validate the efficacy of positive urine cytology as an independent predictor of the presence of LVI, although several reports suggest a significant relationship between positive urine cytology and the presence of LVI $(30,32)$. Despite these limitations, our data suggests that a high plasma fibrinogen level can be an independent prognosticator for worse CSS and ERFS in patients with UTUC undergoing RNU.

In conclusion, stratifying preoperative risk using fibrinogen levels, positive urine cytology, and the presence of hydronephrosis before RNU can provide additional information aboutthe possibility of worse CSS and ERFS rates in patients with localized UTUC. This finding can serve as a basis for selecting candidates for additional therapy before and/or after RNU in patients with UTUC.

\section{Acknowledgements}

Not applicable.

\section{Funding}

No funding was received.

\section{Availability of data and materials}

The datasets used and/or analyzed during the current study are available from the corresponding author on reasonable request.

\section{Authors' contributions}

KK, ST, JA, AH and KI were involved in the conception and design of the study. KK collected and analyzed the data. KK drafted the manuscript. The authenticity of all the raw data was assessed by KK and KI. KK and KI reviewed and edited the manuscript. All authors read and approved the final manuscript.

\section{Ethics approval and consent to participate}

All procedures performed in this study were approved bythe National Defense Medical College (Saitama, Japan; approval no. 2734). All procedures were conducted in accordance with the 1964 Declaration of Helsinki and its later amendments.

\section{Patient consent for publication}

Not applicable.

\section{Competing interests}

The authors declare that they have no competing interests.

\section{References}

1. Roupret M, Babjuk M, Comperat E, Zigeuner R, Sylvester RJ, Burger M, Cowan NC, Böhle A, Van Rhijn BW, Kaasinen E, et al: European association of urology guidelines on upper urinary tract urothelial cell carcinoma: 2015 update. Eur Urol 68: 868-879, 2015.

2. Siegel RL, Miller KD and Jemal A: Cancer statistics, 2019. CA Cancer J Clin 69: 7-34, 2019.

3. Liu JY, Dai YB, Zhou FJ, Long Z, Li YH, Xie D, Liu B, Tang J, Tan J, Yao K and He LY: Laparoscopic versus open nephroureterectomy to treat localized and/or locally advanced upper tract urothelial carcinoma: Oncological outcomes from a multicenter study. BMC Surg 17: 17, 2017.

4. Templeton AJ, Ace O, McNamara MG, Al-Mubarak M, Vera-Badillo FE, Hermanns T, Seruga B, Ocaña A, Tannock IF and Amir E: Prognostic role of platelet to lymphocyte ratio in solid tumors: A systematic review and meta-analysis. Cancer Epidemiol Biomarkers Prev 23: 1204-1212, 2014.

5. Teng JJ, Zhang J, Zhang TY, Zhang S and Li BS: Prognostic value of peripheral blood lymphocyte-to-monocyte ratio in patients with solid tumors: A meta-analysis. Onco Targets Ther 9: 37-47, 2015.

6. Koh CH, Bhoo-Pathy N, Ng KL, Jabir RS, Tan GH, See MH, Jamaris S and Taib NA: Utility of pre-treatment neutrophil-lymphocyte ratio and platelet-lymphocyte ratio as prognostic factors in breast cancer. Br J Cancer 113: 150-158, 2015.

7. Gunduz S, Mutlu H, Tural D, Yildiz O, Uysal M, Coskun HS and Bozcuk H: Platelet to lymphocyte ratio as a new prognostic for patients with metastatic renal cell cancer. Asia Pac J Clin Oncol 11: 288-292, 2015.

8. Allin KH and Nordestgaard BG: Elevated C-reactive protein in the diagnosis, prognosis, and cause of cancer. Crit Rev Clin Lab Sci 48: 155-170, 2011.

9. Kang M, Jeong CW, Kwak C, Kim HH and Ku JH: Preoperative neutrophil-lymphocyte ratio can significantly predict mortality outcomes in patients with non-muscle invasive bladder cancer undergoing transurethral resection of bladder tumor. Oncotarget 8: 12891-12901, 2016.

10. Yuksel OH, Akan S, Uukmez A, Yildirim C, Sahin A and Verit A: Preoperative Glasgow prognostic score as a predictor of primary bladder cancer recurrence. Mol Clin Oncol 5: 201-206, 2016.

11. Yamashita H, Kitayama J, Kanno N, Yatomi Y and Nagawa H: Hyperfibrinogenemia is associated with lymphatic as well as hematogenous metastasis and worse clinical outcome in T2 gastric cancer. BMC Cancer 6: 147, 2006.

12. Yamashita H, Kitayama J and Nagawa H: Hyperfibrinogenemia is a useful predictor for lymphatic metastasis in human gastric cancer. Jpn J Clin Oncol 35: 595-600, 2005.

13. Palumbo JS, Potter JM, Kaplan LS, Talmage K, Jackson DG and Degen JL: Spontaneous hematogenous and lymphatic metastasis, but not primary tumor growth or angiogenesis, is diminished in fibrinogen-deficient mice. Cancer Res 62: 6966-6972, 2002.

14. Fan S, Guan Y, Zhao G and An G: Association between plasma fibrinogen and survival in patients with small-cell lung carcinoma. Thorac Cancer 9: 146-151, 2018.

15. Pichler M, Hutterer GC, Stojakovic T, Mannweiler S, Pummer K and Zigeuner R: High plasma fibrinogen level represents an independent negative prognostic factor regarding cancer-specific, metastasis-free, as well as overall survival in a European cohort of non-metastatic renal cell carcinoma patients. Br J Cancer 109: 1123-1129, 2013.

16. Erdem S, Amasyali AS, Aytac O, Onem K, Issever $\mathrm{H}$ and Sanli O: Increased preoperative levels of plasma fibrinogen and d dimer in patients with renal cell carcinoma is associated with poor survival and adverse tumor characteristics. Urol Oncol 32: 1031-1040, 2014.

17. Sobin LH, Gospodarowicz MK and Wittekind Ch (eds): TNM Classification of Malignant Tumours, 7th Edition. Wiley-Blackwell, Hoboken, NJ, 2009.

18. The International Agency for Research on Cancer (IARC): Pathology and Genetics of Tumours of the Urinary System and Male Genital Organs (IARC WHO Classification of Tumours) 1st Edition. Eble J, Epstein J, Sesterhenn I and Sauter G (eds). World Health Organization, Geneva, 2004. 
19. Qu J, Qu X, Li Z, Zhang Jd, Liu J, Teng Ye, Jin B, Zhao Mf, Yu P, Shi J, et al: Prognostic model based on systemic inflammatory response and clinicopathological factors to predict outcome of patients with node-negative gastric cancer. PLoS One 10 $0128540,2015$.

20. Tanaka N, Kikuchi E, Matsumoto K, Hayakawa N, Ide H, Miyajima A, Nakamura S and Oya M: Prognostic value of plasma fibrinogen levels in patients with localized upper tract urothelial carcinoma. BJU Int 111: 857-864, 2013.

21. Kasuga J, Kawahara T, Takamoto D, Fukui S, Tokita T, Tadenuma T, Narahara M, Fusayasu S, Terao H, Izumi K, et al: Increased neutrophil-to-lymphocyte ratio is associated with disease-specific mortality in patients with penile cancer. BMC Cancer 16: 396, 2016.

22. Uemura K, Kawahara T, Yamashita D, Jikuya R, Abe K, Tatenuma T, Yokomizo Y, Izumi K, Teranishi JI, Makiyama K, et al: Neutrophil-to-lymphocyte ratio predicts prognosis in castration-resistant prostate cancer patients who received cabazitaxel chemotherapy. Biomed Res Int 2017: $7538647,2017$.

23. Lee JH, Ryu KW, Kim S and Bae JM: Preoperative plasma fibrinogen levels in gastric cancer patients correlate with extent of tumor. Hepatogastroenterology 51: 1860-1863, 2004.

24. HKitayama JY, Hatano K, Tsuno N, Osada T, Watanabe T, Tsuruo T, Muto T and Nagawa H: Clustered cancer cells show a distinct adhesion behavior from single cell form under physiological shear conditions. J Exp Clin Cancer Res 20: 407-412, 2001.

25. Çalışkan S and Sungur M: Fibrinogen and D-dimer levels in prostate cancer: Preliminary results. Prostate Int 5: 110-112, 2017.

26. Palumbo JS, Kombrinck KW, Drew AF, Grimes TS, Kiser JH, Degen JL and Bugge TH: Fibrinogen is an important determinant of the metastatic potential of circulating tumor cells. Blood 96 3302-3309, 2000.

27. Kuroda K, Asakuma J, Horiguchi A, Kawaguchi M, Shinchi M, Masunaga A, Tasaki S, Sato A and Ito K: Chronic kidney disease and positive surgical margins as prognosticators for upper urinary tract urothelial carcinoma patients undergoing radical nephroureterectomy. Mol Clin Oncol 10: 547-554, 2019.
28. Bagoly Z: Cancer and thrombosis: A fresh look at an old story. Thromb Res 136: 1-2, 2015.

29. Thurner EM, Krenn-Pilko S, Langsenlehner U, Stojakovic T, Pichler M, Gerger A, Kapp KS and Langsenlehner T: The association of an elevated plasma fibrinogen level with cancer-specific and overall survival in prostate cancer patients. World J Urol 33: $1467-1473,2015$

30. Sakano S, Inamoto $T$, Inoue $R$, Matsumoto $H$, Nagao $K$, Yamamoto Y, Azuma $\mathrm{H}$ and Matsuyama $\mathrm{H}$ : Positive voided urine cytology predicts worse pathological findings of nephroureterectomy specimens in patients with upper tract urothelial carcinoma: Does selective ureteral cytology have an additional efficacy? Jpn J Clin Oncol 45: 968-972, 2015

31. Ito Y, Kikuchi E, Tanaka N, Miyajima A, Mikami S, Jinzaki M and Oya M: Preoperative hydronephrosis grade independently predicts worse pathological outcomes in patients undergoing nephroureterectomy for upper tract urothelial carcinoma. J Urol 185: 1621-1626, 2011.

32. Tanaka N, Kikuchi E, Kanao K, Matsumoto K, Shirotake S, Kobayashi H, Miyazaki Y, Ide H, Obata J, Hoshino K, et al: The predictive value of positive urine cytology for outcomes following radical nephroureterectomy in patients with primary upper tract urothelial carcinoma: A multi-institutional study. Urol Oncol 32: e19-e26, 2014

33. Kohada Y, Hayashi T, Goto K, Kobatake K, Abdi H, Honda Y, Sentani K, Inoue S, Teishima J, Awai K, et al: Preoperative risk classification using neutrophil-lymphocyte ratio and hydronephrosis for upper tract urothelial carcinoma. Jpn J Clin Oncol 48: 841-850, 2018.

34. Chung PH, Krabbe LM, Darwish OM, Westerman ME, Bagrodia A, Gayed BA, Haddad AQ, Kapur P, Sagalowsky AI, Lotan Y and Margulis V: Degree of hydronephrosis predicts adverse pathological features and worse oncologic outcomes in patients with high-grade urothelial carcinoma of the upper urinary tract. Urol Oncol Semin Orig Investig 32: 981-988, 2014.

This work is licensed under a Creative Commons Attribution-NonCommercial-NoDerivatives 4.0 International (CC BY-NC-ND 4.0) License. 